\title{
Sex differences in myelin-associated protein levels within and density of projections between the orbital frontal cortex and dorsal striatum of adult rats: Implications for inhibitory control
}

\author{
Daniel W. Bayless, ${ }^{\mathrm{a},}$ and Jill M. Daniel ${ }^{\mathrm{a}, \mathrm{b}}$ \\ ${ }^{\mathrm{a}}$ Department of Psychology and ${ }^{\mathrm{c}}$ Neuroscience Program, Tulane University, \\ New Orleans, LA 70118, USA
}

\section{Email Addresses: \\ Daniel Bayless: daniel.bayless@ucsf.edu Jill Daniel: jmdaniel@tulane.edu}

*Corresponding Author:

Dr. Daniel W. Bayless

Department of Anatomy

University of California San Francisco

San Francisco, CA 94158

Phone: 1-415-514-4383

Fax: 1-415-514-4360

Email: daniel.bayless@ucsf.edu 


\begin{abstract}
Impulsive actions and decisions often lead to undesirable outcomes. Lesion and neuroimaging studies have revealed that the orbital frontal cortex (OFC) and dorsal striatum (dSTR) play key roles in inhibitory control. It has been proposed that greater OFC input into the dSTR reflects enhanced top-down cognitive control and less impulsive responding. We previously reported a sex difference in inhibitory control, such that female rats make fewer impulsive errors than do male rats. The goal of the present study was to investigate differences in the OFC and dSTR of young adult male and female rats. In Experiment 1, we measured levels of two myelin-associated proteins, myelin basic protein (MBP) and myelin proteolipid protein (PLP), in the OFC and dSTR. Western blot data revealed that females had significantly higher levels of both MBP and PLP in the OFC but similar levels in the dSTR as compared to males. In Experiment 2, we infused the anterograde tracer, biotinylated dextran amine (BDA), into the OFC and measured the density of BDA in the dSTR. BDA was visualized using histochemistry followed by light microscopy imaging and densitometry analysis. Density of BDA in the dSTR was significantly greater in females as compared to males indicating that the projections from the OFC to dSTR may be greater in females as compared to males. Our results suggest a potential neuroanatomical sex difference that may contribute to the reported differences in inhibitory control levels of male and female rats.
\end{abstract}

Key words: sex difference; orbital frontal cortex; striatum; myelin; anterograde tracer; inhibitory control 


\section{Introduction}

Inhibitory control is vital for normal everyday functioning, and deficits in inhibitory control contribute to many neuropsychiatric disorders (Breedlove et al. 2007; Steel and Blaszczynski 1998). Studies investigating the neuroanatomical circuitry of impulsivity have demonstrated the important role that the prefrontal cortex (PFC) plays in inhibitory control in humans and animals (Brass and von Cramon 2002; Cardinal 2006; Dove et al. 2000; Eagle and Baunez 2010). Human subjects with damage to the PFC display impulsive actions during stopsignal tasks (Aron et al. 2003) and impulsive choices during gambling tasks (Bechara et al. 1994). It has been proposed that the PFC influences impulsivity by modulating the operations of lower brain areas involved in reward-based behaviors, such as the striatum (Galvan et al. 2006; Peper et al. 2012; Perry et al. 2011). The striatum is involved in reward associated stimulusresponse behaviors (Eichenbaum 2012). In this view, the PFC acts as a top-down modulator of these lower brain areas, integrating behaviorally relevant information and preventing the overreliance on fixed action patterns (Perry et al. 2011; Peters and Buchel 2011). Using functional magnetic resonance imaging (fMRI), the striatum has been shown to be hyper-responsive when individuals choose immediate small rewards over delayed large rewards (McClure et al. 2004), and the magnitude of striatal activation correlates with the amount of impulsive choices individuals make during a delay-discounting task (Hariri et al. 2006). In addition, using transcranial magnetic stimulation (TMS) to disrupt the PFC leads to in an increase in preference for immediate small rewards over delayed large rewards (Figner et al. 2010), suggesting that blocking the ability of the PFC to modulate the operations of lower brain areas results in increased impulsivity. Furthermore, results using tract-based diffusion tensor imaging have revealed that lower integrity within the frontostrial white matter tract predicts a greater increase 
in impulsivity as the delay for a large reward over an immediate small reward increases (Peper et al. 2012).

Studies investigating sex differences in inhibitory control and the symptomology of attention deficit hyperactivity disorder (ADHD) indicate that males display greater inhibitory control deficits and are more frequently diagnosed with the hyperactive-impulsive subtype of ADHD than are females (Davies 2014; Gershon 2002; Rucklidge 2010). In addition, many but not all studies examining inhibitory control in the general population indicate that males are more impulsive than are females (Fillmore and Weafer 2004; Heyman and Gibb 2006; Kirby and Marakovic 1996; Li-Grining 2007; Moilanen et al. 2009; Reynolds et al. 2006; Rosenblitt et al. 2001; Skinner et al. 2004; Van et al. 2008; Whiteside and Lynam 2003). Our laboratory and others have reported that adult male rats make more impulsive actions than do females when a delayed response is required during the 5-choice serial reaction time task and other tests of spatial divided attention (Bayless et al. 2012; Jentsch and Taylor 2003). In addition, our laboratory has demonstrated that prepubertal male rats make more impulsive choices for an immediate small food reward over a delayed large food reward than do females and that this sex difference is organized by neonatal exposure to estrogens and androgens (Bayless et al. 2013).

One possible neuroanatomical mechanism that could contribute to a sex difference in inhibitory control would be a difference in myelination and density of projections from the orbital frontal cortex (OFC) to dorsal striatum (dSTR), such that females as compared to males have increased OFC input into the dSTR leading to enhanced inhibitory control. The levels of myelination or white matter vary across brain regions, and sex differences in brain connectivity are reported (For review see, Gong et al. 2011). To our knowledge no study to date has examined sex differences in myelination and projections from the OFC to dSTR. Projections from the dSTR diverge into two routes: the direct pathway leading to excitation of thalamic projections to 
motor cortex and the indirect pathway leading to inhibition of thalamic projections to motor cortex (Graybiel 2000; Mink 1996). The balance between these two pathways allows for the release of desired behavioral patterns while inhibiting undesired behavioral patterns (Miller and Buschman 2007). The excitatory glutamatergic inputs of the OFC into the dSTR are hypothesized to modulate the activity of the direct and indirect pathways of the dSTR (For review see, Eagle and Baunez 2010). A neuroanatomical sex difference in the density of projections from the OFC to dSTR in female rats as compared to males could provide females with increased OFC input into the dSTR leading to increased inhibition of thalamic projections to motor cortex via the indirect pathway, thereby dampening motor output and enhancing the ability of females to inhibit undesirable behaviors.

The goal of the present study was to test the hypothesis that there is a sex difference in male and female rats such that myelination and density of projections from the OFC to dSTR are greater in females as compared to males. In Experiment 1, we examined sex differences in the levels of myelin-associated proteins in the OFC and dSTR via western blotting for myelin basic protein (MBP) and myelin proteolipid protein (PLP). MBP is responsible for the adhesion of multilayered compact myelin to axons and to itself and is the second most abundant protein in central nervous system (CNS) myelin (Boggs 2006). PLP is a hydrophobic integral membrane protein and the most abundant protein in CNS myelin (Greer and Lees 2002). Increased levels of MBP and PLP are indicative of increased levels of myelination. In Experiment 2, in order to gain insight into a possible sex difference in the density of the projections from the OFC to dSTR, we infused an anterograde tracer into the OFC of male and female rats and measured the density of the tracer in the dSTR. 


\section{Experimental Procedures}

\subsection{Experiment 1: Analysis of myelin-associated protein levels in the OFC and dSTR}

\subsubsection{Animals}

Nine female and eight male Long-Evans hooded rats, approximately 60 days old, were purchased from Harlan Sprague-Dawley. Animal care was in accordance with the guidelines set by the National Institutes of Health Guide for the Care and Use of Laboratory Animals and all procedures were approved by the Institutional Animal Care and Use Committee of Tulane University. Rats were housed in same-sex pairs in a temperature controlled vivarium under a 12h light/dark cycle (lights on at 7:00 a.m.). Prior to use in the present experiment, rats were used to pilot behavioral tasks for future use in the laboratory.

\subsubsection{Vaginal Cytology}

To control for effects of fluctuating ovarian hormones, vaginal smears of female rats were collected by lavage each morning and analyzed daily beginning two weeks prior to brain dissection. To control for handling effects, males underwent sham smears during which a small amount of water was placed on the genitals using a medicine dropper. Females were sacrificed at the proestrous stage of the estrous cycle, at which time circulating estradiol levels are at their peak and vaginal cytology is characterized by large nucleated epithelial cells (Becker et al. 2005). Each male was paired with a particular female and was sacrificed on the same day as that female. 


\subsubsection{Tissue dissection and processing}

At approximately 85 days old, male and female rats were deeply anesthetized by intraperitoneal injection of ketamine (100 mg/kg) and xylazine $(7 \mathrm{mg} / \mathrm{kg})$ and killed by decapitation. Whole brains were removed and quick-frozen on dry ice. In a cryostat at $-20^{\circ} \mathrm{C}$, coronal sections containing the OFC and dSTR were cut using coordinates from Paxinos and Watson 1998 (OFC: AP +4.2 mm to +2.7 mm; dSTR: AP +1.7 mm to -0.4 mm). Using a scalpel and visual cues from natural boundaries such as the corpus callosum, the OFC and dSTR were dissected (as described in Spijker 2011). Tissue samples from both hemispheres were pooled for each animal and stored at $-80^{\circ} \mathrm{C}$ until processing. Tissue was homogenized in $20 \mu \mathrm{l} / \mathrm{mg}$ lysis buffer containing 1mM EGTA, 1mM EDTA, 20 mM Tris, 1 mM sodium pyrophosphate tetrabasic decahydrate, $4 \mathrm{mM}$ 4-nitrophenyl phosphate disodium salt hexahydrate, $0.1 \mu \mathrm{M}$ microcystin, and 1\% protease inhibitor cocktail (Sigma-Aldrich). Samples were then centrifuged for $15 \mathrm{~min}$ at $1000 \mathrm{x} \mathrm{g}$ at $4 \mathrm{C}$, protein concentration of supernatants was determined (Bradford Protein Assay Kit; Pierce, Rockford, IL), and each sample was diluted 1:1 with Laemmli Sample Buffer (Bio-Rad; Hercules, CA), mixed with $350 \mathrm{mM}$ D,L-dithiothreitol, boiled for 5 min, and stored at $-80 \mathrm{C}$.

\subsubsection{Proteins of interest}

Western blotting procedures were used to assess protein levels of MBP and PLP in the OFC and dSTR. MBP consists of four major isoforms with molecular masses of 21.5, 18.5, 17.0, and $14.0 \mathrm{kDa}$ (Akiyama et al. 2002). PLP consists of one major isoform with a molecular mass of $23 \mathrm{kDA}$ (Greer and Lees 2002). 


\subsubsection{Electrophoresis and immunostaining}

For each MBP and PLP sample, $25 \mu \mathrm{g}$ of total protein was loaded and separated at $200 \mathrm{~V}$ on 15\% SDS-PAGE gels (Bio-Rad) for 60 min. Molecular weight markers (Kaleidoscope; BioRad) were included with each run. Proteins were transferred to nitrocellulose membranes at 100V for 60 min. Membranes were blocked with 5\% nonfat dry milk in 0.1\% Tween/1 X Trisbuffered saline (TTBS) at room temperature for $60 \mathrm{~min}$. Following this, membranes were cut to separate the section containing the proteins of interest from the section containing the loading control $\beta$-actin. Membranes were then incubated with primary antibodies for MBP (mouse monoclonal; 1:5000, Abcam, AB78156), PLP (mouse monoclonal; 1:1500, Millipore, MAB388), or $\beta$-actin (mouse monoclonal; 1:15,000; Santa Cruz) overnight at $4 \mathrm{C}$ in $1 \%$ nonfat dry milkTTBS. Blots were washed three times for 15 min each with TTBS and incubated with 5\% nonfat dry milk containing goat antimouse IgG (MBP, 1:10,000; PLP, 1:10,000; $\beta$-actin, 1:10,000; Santa Cruz) conjugated to horseradish peroxidase for $1.5 \mathrm{~h}$ at room temperature. Blots were washed again three times for 15 min each and incubated for 1 min with the chemiluminescent substrate Pierce ECL western blotting substrate (MBP; $\beta$-actin; Fisher Scientific) or 5 min with the chemiluminescent substrate SuperSignal West Femto (PLP; Fisher Scientific) and exposed to film (Kodak Biomax MR) for varying durations to capture optimal signal intensity. Films were imaged using MCID Core imaging software (InterFocus Imaging Ltd., Cambridge, England), and optical density x area was measured for bands of interest. All values were represented as a percentage relative to $\beta$-actin for each sample.

\subsubsection{Statistical analyses}

For PLP data, the optical density $\mathrm{x}$ area values as a percentage of $\beta$-actin loading control were analyzed using a one-way ANOVA. For MBP data, the optical density x area values as a 
percentage of $\beta$-actin loading control were analyzed using a repeated measures ANOVAs with isoform as the within-subjects factor and sex as the between-subjects factor.

\subsection{Experiment 2: Analysis of projections from the OFC to dSTR}

\subsubsection{Animals}

Six male and six female Long-Evans hooded rats, approximately 60 days old, were purchased from Harlan Sprague Dawley Inc. (Indianapolis, IN). Rats were housed in same-sex pairs, and animal care was the same as described in Experiment 1.

\subsubsection{Stereotaxic surgery and tracer injection}

A week after arrival, rats underwent stereotaxic surgery to allow for bilateral microinjection of the anterograde tracer, biotinylated dextran amine (BDA), into the OFC. BDA is a widely used anterograde tracer that is selectively taken up by neurons at the site of injection (Wang et al. 2013). BDA is well transported both retrogradely and anterogradely depending on its molecular weight (Lazarov 2013). At $10 \mathrm{kDa}$, as was used in the current experiment, BDA is anterogradely transported. BDA is biotinylated (Reiner et al. 2000). Thus, there is no need to attach a biotin-conjugated secondary to BDA in order to visual a peroxidase reaction product after incubation with avidin biotin peroxidase complex (ABC) followed by diaminobenzidine (DAB) during histochemistry (Lazarov 2013). Anterograde tracer and histochemistry techniques

were based upon Springer Protocols for anterograde tracing experiments (Lazarov 2013; Wang et al. 2013).

Surgeries were conducted on anesthetized rats using appropriate aseptic techniques. Rats were anesthetized by intraperitoneal injection of ketamine $(100 \mathrm{mg} / \mathrm{kg})$ and xylazine $(7 \mathrm{mg} / \mathrm{kg})$. 
Buprenorphine (0.025 mg/kg, Buprenex) was administered subcutaneously as an analgesic. Rats were placed in a stereotaxic apparatus. An incision was made along the midline of the head of each rat, and the underlying fascia was scraped to the side. The local anesthetic, $0.5 \%$ lidocaine was applied to the fascia at the time of the incision. Using a small drill mounted on a stereotaxic arm, a 0.9 diameter hole was drilled through the skull. Using a Hamilton syringe, $0.5 \mu \mathrm{l}$ per hemisphere of $10 \% \mathrm{BDA}(10 \mathrm{kDa})$ in aCSF were infused bilaterally into the OFC as shown in Figure 1 ( $\mathrm{AP}+3.2 \mathrm{~mm}, \mathrm{ML} \pm 2.5 \mathrm{~mm}$ from bregma, and $\mathrm{DV}-4.6 \mathrm{~mm}$ from the dura, coordinates from Paxinos and Watson 1998). The skull surface was completely dried, and the entire infusion site was covered with sterile bone wax. The incision was closed with sterile absorbable sutures.

\subsubsection{Perfusion and fixation}

After a survival period of 10 days allowing for anterograde transport of the tracer to the dSTR, rats were deeply anesthetized by intraperitoneal injection of ketamine $(100 \mathrm{mg} / \mathrm{kg})$ and xylazine $(7 \mathrm{mg} / \mathrm{kg})$. The stage of the estrous cycle after the 10 day survival period was random across females. The chest and abdominal cavity was surgically opened. Rats were injected intracardially with $0.05 \mathrm{ml}$ of heparin, an anticoagulant. Rats were then perfused intracardially with $200 \mathrm{ml}$ of $0.9 \%$ saline followed by $500 \mathrm{ml}$ of $4 \%$ paraformaldehyde in $0.1 \mathrm{M}$ phosphate buffered saline (PBS). After perfusion, brains were removed and post-fixed in a fresh fixative of $30 \%$ sucrose in $0.1 \mathrm{M}$ PBS.

\subsubsection{Tissue sectioning}

Frozen $50 \mu \mathrm{m}$ coronal sections of the OFC from AP $+4.2 \mathrm{~mm}$ to $+2.7 \mathrm{~mm}$ and dSTR from $\mathrm{AP}+1.7 \mathrm{~mm}$ to $-0.4 \mathrm{~mm}$ were collected in cyroprotectant (1\% polyvinylpyrrolidone, $30 \%$ sucrose, 30\% ethylene glycol in $0.1 \mathrm{M}$ PBS) using a sliding microtome. Sections were store in 
$0.5 \mathrm{ml}$ vials in cyroprotectant at $4^{\circ} \mathrm{C}$ until further use. OFC sections at $\mathrm{AP}+3.2 \mathrm{~mm}$ were mounted onto gelatin-coated slides and stained with cresyl violet to verify infusion location (See Figure 1). Three consecutive dSTR sections at AP $+0.5 \mathrm{~mm}$ were used for imaging and statistical analysis of BDA density.

\subsubsection{Histochemistry}

Sections were washed three times for 10 min each in 0.05M PBS and then incubated for 60 min in $0.1 \%$ bovine serum albumin in $0.05 \mathrm{M}$ PBS to block non-specific binding sites. Following blocking, sections were washed three times for 10 min each in 0.05M PBS. Sections were then incubated for 120 mins in avidin biotin peroxidase complex (ABC) reagent (Vectastain Elite Kit, Vector Laboratories, Inc.) in 0.3\% Triton X in 0.05M PBS. Following three more washes for 10 min each in 0.05M PBS, the peroxidase reaction product was visualized by incubation in a solution containing 0.05\% diaminobenzidine (DAB; Sigma Aldrich) and 0.01\% $\mathrm{H}_{2} \mathrm{O}_{2}$ in $0.1 \mathrm{M}$ PBS for 7 min. Sections were then wash again three times for $10 \mathrm{~min}$ in $0.01 \mathrm{M}$ PBS, mounted onto gelatin-coated slides, and allowed to air-dry overnight. The following day, the slides were placed in xylene for 5 min, and then coverslipped using diluted permount.

\subsubsection{Imaging and quantification of BDA density}

Sections were imaged at 20x magnification using a light microscope (Olympus IX71) and digital camera (Hamamatsu Camera; 0.1 s exposure) interfaced with HC Image Software. MCID Analysis software was used to calibrate images to control for variations in background density. Optical density was measured within circular regions (1 mm diameter) of the dorsolateral striatum (dlSTR) determined by measuring $0.6 \mathrm{~mm}$ horizontally and $0.75 \mathrm{~mm}$ vertically from the corpus callosum and within circular regions (1 mm diameter) of the dorsomedial striatum 
(dmSTR) determined by measuring $0.6 \mathrm{~mm}$ horizontally from the lateral ventricle and $1 \mathrm{~mm}$ vertically from the corpus callosum (See Figure 2). Figure 3 provides example images of anterograde tracer staining in the dSTR at varying magnification levels.

\subsubsection{Statistical analyses}

Three consecutive sections at AP $+0.5 \mathrm{~mm}$ for each rat were imaged and optical density values were averaged for statistical analysis of BDA density. The optical density values as a percentage of average female density for the left and right hemispheres of the dISTR and dmSTR were analyzed using a repeated measures ANOVA with hemisphere as the within-subjects factor and sex as the between-subjects factor for each brain region.

\section{Results}

\subsection{Experiment 1: Analysis of myelin-associated protein levels in the OFC and dSTR}

\subsubsection{MBP in OFC and dSTR}

Western blots for MBP revealed four isoform bands of MBP-like immunoreactivity at approximately 21.5, 18.5, 17.0, and 14.0 kDa. As illustrated in Figure 4A-C, analysis of protein levels of MBP in the OFC of males and females revealed a main effect of MBP isoform $(F(3,45)$ $=51.17, p<0.001)$ and a main effect of $\operatorname{sex}(F(1,15)=4.95, p=0.042)$. There was no interaction between sex and MBP isoform. There was no effect of sex on levels of $\beta$-actin loading control. Results indicate that protein levels of MBP in the OFC are significantly greater in females as compared to males. 
As illustrated in Figure 4D-F, analysis of protein levels of MBP in the dSTR of males and females revealed a main effect of MBP isoform $(F(3,45)=70.26, p<0.001)$, indicating that protein levels varied across the four isoforms. However, there was no main effect of sex or interaction between sex and MBP isoform. There was no effect of sex on levels of $\beta$-actin loading control. Results indicate that protein levels of MBP in the dSTR are not significantly different between males and females.

\subsection{2. $P L P$ in $O F C$ and $d S T R$}

Western blots for PLP revealed a band of PLP-like immunoreactivity at approximately 23 kDa. As illustrated in Figure 5A-B, analysis of protein levels of PLP in the OFC of males and females revealed a main effect of $\operatorname{sex}(F(1,15)=5.40, p=0.035)$. There was no effect of sex on levels of $\beta$-actin loading control. Results indicate that protein levels of PLP in the OFC are significantly greater in females as compared to males.

As illustrated in Figure 5C-D, analysis of protein levels of PLP in the dSTR of males and females revealed no main effect of sex. There was no effect of sex on levels of $\beta$-actin loading control. Results indicate that protein levels of PLP in the dSTR are not significantly different between males and females.

\subsection{Experiment 2: Analysis of projections from the OFC to dSTR}

\subsubsection{Anterograde tracer density in $\operatorname{dISTR}$ and $d m S T R$}

As illustrated in Figure 6A, analysis of optical density of OFC-injected anterograde tracer in the dlSTR of males and females revealed a significant main effect of $\operatorname{sex}(\mathrm{F}(1,10)=5.67, p=$ 0.039), indicating that the density of projections from the OFC to dlSTR is significantly greater 
in females as compared to males. There was no significant main effect of hemisphere, indicating that the density of the tracer was similar in the left and right hemispheres.

As illustrated in Figure 6B, analysis of optical density of OFC-injected anterograde tracer in the dmSTR of males and females revealed a strong trend to significance for a main effect of sex $(F(1,10)=4.57, p=0.058)$, suggesting that the density of projections from the OFC to dmSTR is greater in females as compared to males. There was no significant main effect of hemisphere, indicating that the density of the tracer was similar in the left and right hemispheres.

\section{Discussion}

The present experiments demonstrate that myelin-associated protein levels in the OFC and the density of an OFC-infused anterograde tracer is greater in the dSTR of female rats as compared to male rats. Protein levels of MBP and PLP were significantly greater in the OFC but not the dSTR of females as compared to males, suggesting that myelination is increased in the OFC but similar in the dSTR of females as compared to males. Furthermore, the density of an anterograde tracer injected into the OFC and measured in the dSTR was significantly greater in females as compared to males, indicating that the density of projections from the OFC to dSTR may be greater in females as compared to males. These sex differences are consistent with the possibility that females have increased OFC input into the dSTR leading to greater modulation of the reward-based stimulus-response behaviors in females as compared to males. The current findings suggest a potential neuroanatomical mechanism that could underlie the previously reported sex difference in inhibitory control (Bayless et al. 2013; Bayless et al. 2012; Jentsch and Taylor 2003; Kirby and Marakovic 1996; Li-Grining 2007; Moilanen et al. 2009; Rosenblitt et al. 2001; Van et al. 2008; Whiteside and Lynam 2003). 
The current finding of a sex difference in the levels of myelin-associated proteins in the OFC of rats is consistent with the findings from an MRI study in humans that reported that women have more white matter voxels in frontal and parietal brain areas that correlate with intelligence scores than do men (Haier et al. 2005). In the CNS, myelin is formed by oligodendrocytes that wrap tightly around axons insulating and increasing the speed of neurotransmission (Sherman and Brophy 2005). The ratio of the axon diameter to myelin diameter (g-ratio) is fairly consistent across myelinated neurons, meaning that larger axons have thicker myelin, and vice versa (Sherman and Brophy 2005). Therefore, increased levels of myelination indicate either a greater number of neuronal connections or a greater average axonal diameter of neurons. Both the number of neuronal connections and axonal diameter are associated with faster, more efficient neuronal communication (Sherman and Brophy 2005). Therefore, the current results are consistent with the hypothesis that the speed of neurotransmission within the OFC but not the dSTR is greater in female rats as compared to male rats resulting in increased OFC input into and control over the dSTR in females as compared to males. The corticostriatal projections of interest in the current study are fully myelinated, but interestingly, the dopaminergic inputs into the dSTR from the substantia nigra are mostly unmyelinated (Pissadaki and Bolam 2013). Our western blot assays of myelinassociated protein levels in the OFC and dSTR do not provide insight into the density of projections from the OFC to dSTR. Therefore in the current experiment, we measured density levels of an OFC-infused anterograde tracer in the dSTR resulting in the novel discovery that the density of the tracer in the dSTR was greater in females as compared to males. Taken together, the increased myelin-associated protein levels in the OFC and increased anterograde tracer density in the dSTR of females as compared to males indicate that OFC input into the dSTR may be greater in female rats than it is in male rats. 
Projections from the OFC distribute widely throughout the brain, including cortical targets, such as the medial prefrontal, insular, piriform, and parahippocampal cortices, and subcortical targets, such as the dorsal and ventral striatum, septum, amygdala, thalamus, hypothalamus, substantia nigra, ventral tegmental area, and dorsal and median raphe (Hoover and Vertes 2011). The excitatory glutamatergic projections from the OFC to dSTR are strongly implicated in impulse control (For review see, Eagle and Baunez 2010). Studies using the vesicular glutamate transporter type 1 as a marker of cortical innervation indicate that dSTR medium-sized spiny neurons of both the direct and indirect pathway receive cortical input (Doig et al. 2010; Huerta-Ocampo et al. 2014). The balance between the direct and indirect pathways of the dSTR allows for the release of desired behavioral patterns while inhibiting undesired behavioral patterns (Miller and Buschman 2007). The current findings in combination with our previous reports that female rats display increased inhibitory control as compared to males (Bayless et al. 2013; Bayless et al. 2012) support the hypothesis that the OFC influences impulsivity by modulating the operations of lower brain areas involved in reward-based behaviors, such as the dSTR (Galvan et al. 2006; Peper et al. 2012; Perry et al. 2011). The increased density of the OFC-infused anterograde tracer in the dSTR and increased myelinassociated protein levels in the OFC in female rats as compared to males may allow for increased OFC control over the dSTR leading to increased inhibition of thalamic projections to motor cortex via the indirect pathway, thereby dampening motor output and enhancing the ability of females to inhibit undesirable behaviors.

The time course of many neurodevelopmental processes varies between the sexes (For review see, Brenhouse and Andersen 2011). In the current study, animals were age-matched and approximately 85 days of age at the time of the experiments. The onset of puberty is typically around 35 days of age for female rats and 45 days of age for male rats (Kennedy and Mitra 
1963). Although the animals in the current study are considered adults, some neurodevelopmental processes are still ongoing in young adulthood (Brenhouse and Andersen 2011). Therefore, it is possible that a developmental delay in the maturation of OFC projections to the dSTR in males as compared to females could contribute to the neuroanatomical sex differences reported here. Future studies are needed to fully understand the impact that developmental sex differences might have on OFC projections to the dSTR and inhibitory control. In addition, in the current study our focus was on the mechanisms of inhibitory control associated with the dSTR. The ventral striatum (vSTR) is strongly implicated in reward seeking behavior (Dalley et al. 2008) and could also contribute to differences in impulsivity in males and females. Future research examining the vSTR in males and females will further enhance our understanding of the neurobiological underpinnings of the reported sex differences in impulsivity. Furthermore, the current study only provides a neuroanatomical sex difference and does not provide an isolated assessment of the OFC inputs into the direct or indirect pathway of the dSTR. Future studies are needed to demonstrate the causal link between the possible sex difference in density of projections from the OFC to dSTR and enhanced inhibitory control.

Previous research in our laboratory has demonstrated that the sex difference in impulsive choice behavior in prepubertal rats is organized neonatally by the actions of both androgens and estrogens (Bayless et al. 2013). It is unclear if the sex differences in myelin-associated proteins in the OFC and density of the OFC-infused anterograde tracer in the dSTR are organized by neonatal hormone levels. Future studies are needed to determine if this is the case. However, it is possible that epigenetic changes caused by neonatal activation of estrogen or androgen receptors could cause changes in the rate of apoptotic cell death during development that might produce the sex difference in the number of cells that project from the OFC to dSTR. Sex differences in cell number could be established through neurogenesis, neuronal migration, phenotypical 
differentiation, or cell death. Of these neurodevelopmental events, cell death has received the majority of the support as the most common mechanism by which sex differences in cell number are established (Forger 2009). Cell death is a naturally-occurring and essential process in the brain, and over 50\% of neurons born during neurodevelopment undergo apoptosis or programmed cell death during development (Oppenheim 1991). Expression of high levels of the Bax protein promotes apoptosis, whereas expression of high levels of the $\mathrm{Bcl}-2$ protein protects neurons from apoptosis (Forger 2009). Neonatal activation of estrogen or androgen receptors could affect the development of impulsive behavior by increasing histone acetyltransferase activity at the promoter region of the Bax gene in neurons projecting from the OFC to dSTR during neonatal development in males resulting in increased gene transcription of the Bax protein and ensuing cell death of these neurons producing an increased impulsive phenotype later in life. Deletion of the Bax gene eliminates the sex difference in cell number in some sexually dimorphic brain areas (Forger et al. 2004; Gotsiridze et al. 2007), and the human Bcl-2 gene contains the sequence for the estrogen-response element (Perillo et al. 2000). So, it is possible that neonatal activation of estrogen receptors could alter the rate of cell death in specific brain areas in a sex-dependent manner. Future studies are needed to test this hypothesis and investigate the organizational role of neonatal estrogen and androgen levels in the neurodevelopmental basis of the sex differences reported here.

\subsection{Conclusions}

Collectively, the present experiments demonstrate that female rats as compared to males have increased levels of myelin-associated proteins in the OFC and an increased density of an OFC-infused anterograde tracer in the dSTR. These novel neuroanatomical sex differences could underlie the reported differences in inhibitory control levels of male and female rats by providing 
females as compared to males with increased OFC input into the dSTR leading to greater modulation of the reward-based stimulus-response behaviors of the dSTR. In addition, the sex differences reported here have implications for the understanding of sex differences in impulsivity in the general population as well as in neuropsychiatric disorders, such as ADHD, pathological gambling, and drug addiction.

\section{Acknowledgements}

This work was supported by the National Science Foundation Grant 0951008 to JMD and State of Louisiana Board of Regents Graduate Fellowship LEQSF(2009-2014)-GF-13 to DWB. 


\section{Reference List}

Akiyama, K., Ichinose, S., Omori, A., Sakurai, Y., and Asou, H. 2002. Study of expression of myelin basic proteins (MBPs) in developing rat brain using a novel antibody reacting with four major isoforms of MBP. J. Neurosci. Res. 68: 19-28.

Aron, A.R., Fletcher, P.C., Bullmore, E.T., Sahakian, B.J., and Robbins, T.W. 2003. Stop-signal inhibition disrupted by damage to right inferior frontal gyrus in humans. Nat. Neurosci. 6: 115-116.

Bayless, D.W., Darling, J.S., and Daniel, J.M. 2013. Mechanisms by which neonatal testosterone exposure mediates sex differences in impulsivity in prepubertal rats. Horm. Behav. 64: 764-769.

Bayless, D.W., Darling, J.S., Stout, W.J., and Daniel, J.M. 2012. Sex differences in attentional processes in adult rats as measured by performance on the 5-choice serial reaction time task. Behav. Brain Res. 235: 48-54.

Bechara, A., Damasio, A.R., Damasio, H., and Anderson, S.W. 1994. Insensitivity to future consequences following damage to human prefrontal cortex. Cognition 50: 7-15.

Becker, J.B., Arnold, A.P., Berkley, K.J., Blaustein, J.D., Eckel, L.A., Hampson, E., Herman, J.P., Marts, S., Sadee, W., Steiner, M., Taylor, J., and Young, E. 2005. Strategies and methods for research on sex differences in brain and behavior. Endocrinology 146: 16501673.

Boggs, J.M. 2006. Myelin basic protein: a multifunctional protein. Cell Mol. Life Sci. 63: 19451961.

Brass, M. and von Cramon, D.Y. 2002. The role of the frontal cortex in task preparation. Cereb. Cortex 12: 908-914.

Breedlove, S.M., Rosenzweig M.R., and Watson N.V. 2007. Psychopathology: Biological basis of behavioral disorders. In Biological Psychology, pp. 482-494. Sinauer Associates, Sunderland, MA.

Brenhouse, H.C. and Andersen, S.L. 2011. Developmental trajectories during adolescence in males and females: A cross-species understanding of underlying brain changes. Neurosci. \& Biobehav. Rev. 35: 1687-1703.

Cardinal, R.N. 2006. Neural systems implicated in delayed and probabilistic reinforcement. Neural Netw. 19: 1277-1301.

Dalley, J.W., Mar, A.C., Economidou, D., and Robbins, T.W. 2008. Neurobehavioral mechanisms of impulsivity: fronto-striatal systems and functional neurochemistry. Pharmacol. Biochem. Behav. 90: 250-260. 
Davies, W. 2014. Sex differences in Attention Deficit Hyperactivity Disorder: Candidate genetic and endocrine mechanisms. Front Neuroendocrinol. 35: 331-346.

Doig, N.M., Moss, J., and Bolam, J.P. 2010. Cortical and thalamic innervation of direct and indirect pathway medium-sized spiny neurons in mouse striatum. J. Neurosci. 44: 14610 $-14618$

Dove, A., Pollmann, S., Schubert, T., Wiggins, C.J., and von Cramon, D.Y. 2000. Prefrontal cortex activation in task switching: an event-related fMRI study. Brain Res. Cogn Brain Res. 9: 103-109.

Eagle, D.M. and Baunez, C. 2010. Is there an inhibitory-response-control system in the rat? Evidence from anatomical and pharmacological studies of behavioral inhibition. Neurosci. Biobehav. Rev. 34: 50-72.

Eichenbaum, H. 2012. A brain system for procedural memory. In The Cognitive Neuroscience of Memory, pp. 267-289. Oxford University Press, New York.

Figner, B., Knoch, D., Johnson, E.J., Krosch, A.R., Lisanby, S.H., Fehr, E., and Weber, E.U. 2010. Lateral prefrontal cortex and self-control in intertemporal choice. Nat. Neurosci. 13: 538-539.

Fillmore, M.T. and Weafer, J. 2004. Alcohol impairment of behavior in men and women. Addiction 99: 1237-1246.

Forger, N.G. 2009. Control of cell number in the sexually dimorphic brain and spinal cord. $J$. Neuroendocrinol. 21: 393-399.

Forger, N.G., Rosen, G.J., Waters, E.M., Jacob, D., Simerly, R.B., and de Vries, G.J. 2004. Deletion of Bax eliminates sex differences in the mouse forebrain. Proc. Natl. Acad. Sci. U. S. A 101: 13666-13671.

Galvan, A., Hare, T.A., Parra, C.E., Penn, J., Voss, H., Glover, G., and Casey, B.J. 2006. Earlier development of the accumbens relative to orbitofrontal cortex might underlie risk-taking behavior in adolescents. J. Neurosci. 26: 6885-6892.

Gershon, J. 2002. A meta-analytic review of gender differences in ADHD. J. Atten. Disord. 5: 143-154.

Gong, G., He, Y., and Evans, A.C. 2011. Brain connectivity: gender makes a difference. Neuroscientist. 17: 575-591.

Gotsiridze, T., Kang, N., Jacob, D., and Forger, N.G. 2007. Development of sex differences in the principal nucleus of the bed nucleus of the stria terminalis of mice: role of Baxdependent cell death. Dev. Neurobiol. 67: 355-362.

Graybiel, A.M. 2000. The basal ganglia. Curr. Biol. 10: R509-R511. 
Greer, J.M. and Lees, M.B. 2002. Myelin proteolipid protein--the first 50 years. Int. J. Biochem. Cell Biol. 34: 211-215.

Haier, R.J., Jung, R.E., Yeo, R.A., Head, K., and Alkire, M.T. 2005. The neuroanatomy of general intelligence: sex matters. Neuroimage. 25: 320-327.

Hariri, A.R., Brown, S.M., Williamson, D.E., Flory, J.D., de, W.H., and Manuck, S.B. 2006. Preference for immediate over delayed rewards is associated with magnitude of ventral striatal activity. J. Neurosci. 26: 13213-13217.

Heyman, G.M. and Gibb, S.P. 2006. Delay discounting in college cigarette chippers. Behav. Pharmacol. 17: 669-679.

Hoover, W.B. and Vertes, R.P. 2011. Projections of the medial orbital and ventral orbital cortex in the rat. J. Comp. Neurol. 519: 3766-3801.

Huerta-Ocampo, I., Mena-Segovia, J., and Bolam, J.P. 2014. Convergence of cortical and thalamic input to direct and indirect pathway medium spiny neurons in the striatum. Brain Struct Funct. 219:1787-1800.

Jentsch, J.D. and Taylor, J.R. 2003. Sex-related differences in spatial divided attention and motor impulsivity in rats. Behav. Neurosci. 117: 76-83.

Kennedy, G.C. and Mitra, J. 1963. Body weight and food intake as initiating factors for puberty in the rat. J. Physiol 166: 408-418.

Kirby, K.N. and Marakovic, N.N. 1996. Delay-discounting probabilistic rewards: Rates decrease as amounts increase. Psychonomic Bulletin \& Review 3: 100-104.

Lazarov, N.E. 2013. Neuroanatomical tract-tracing using biotinylated dextran amine. Methods Mol. Biol. 1018: 323-334.

Li-Grining, C.P. 2007. Effortful control among low-income preschoolers in three cities: Stability, change, and individual differences. Dev. Psychol. 43: 208-221.

McClure, S.M., Laibson, D.I., Loewenstein, G., and Cohen, J.D. 2004. Separate neural systems value immediate and delayed monetary rewards. Science 306: 503-507.

Miller, E.K. and Buschman, T.J. 2007. Bootstrapping your brain: How interactions between the frontal cortex and basal ganglia may produce organized actions and lofty thoughts. In Neurobiology of Learning and Memory (eds. R.P. Kesner and J.L. Martinez), pp. 339351. Academic Press, Burlington, MA.

Mink, J.W. 1996. The basal ganglia: focused selection and inhibition of competing motor programs. Prog. Neurobiol. 50: 381-425. 
Moilanen, K.L., Shaw, D.S., Dishion, T.J., Gardner, F., and Wilson, M. 2009. Predictors of Longitudinal Growth in Inhibitory Control in Early Childhood. Soc. Dev. 19: 326-347.

Oppenheim, R.W. 1991. Cell death during development of the nervous system. Annu. Rev. Neurosci. 14: 453-501.

Paxinos, G. and Watson, C. 1998. The rat brain in stereotaxic coordinates. Academic Press, San Diego, CA.

Peper, J.S., Mandl, R.C., Braams, B.R., de, W.E., Heijboer, A.C., Koolschijn, P.C., and Crone, E.A. 2012. Delay Discounting and Frontostriatal Fiber Tracts: A Combined DTI and MTR Study on Impulsive Choices in Healthy Young Adults. Cereb. Cortex. 23(7): 16951702.

Perillo, B., Sasso, A., Abbondanza, C., and Palumbo, G. 2000. 17beta-estradiol inhibits apoptosis in MCF-7 cells, inducing bcl-2 expression via two estrogen-responsive elements present in the coding sequence. Mol. Cell Biol. 20: 2890-2901.

Perry, J.L., Joseph, J.E., Jiang, Y., Zimmerman, R.S., Kelly, T.H., Darna, M., Huettl, P., Dwoskin, L.P., and Bardo, M.T. 2011. Prefrontal cortex and drug abuse vulnerability: translation to prevention and treatment interventions. Brain Res. Rev. 65: 124-149.

Peters, J. and Buchel, C. 2011. The neural mechanisms of inter-temporal decision-making: understanding variability. Trends Cogn Sci. 15: 227-239.

Pissadaki, E.K. and Bolam, J.P. 2013. The energy cost of action potential propagation in dopamine neurons: clues to susceptibility in Parkinson's disease. Frontiers in Comp. Neurosci. 7: 1-17.

Reiner, A., Veenman, C.L., Medina, L., Jiao, Y., Del, M.N., and Honig, M.G. 2000. Pathway tracing using biotinylated dextran amines. J. Neurosci. Methods 103: 23-37.

Reynolds, B., Richards, J.B., and de, W.H. 2006. Acute-alcohol effects on the Experiential Discounting Task (EDT) and a question-based measure of delay discounting. Pharmacol. Biochem. Behav. 83: 194-202.

Rosenblitt, J.C., Soler, H., Johnson, S.E., and Quadagno, D.M. 2001. Sensation seeking and hormones in men and women: exploring the link. Horm. Behav. 40: 396-402.

Rucklidge, J.J. 2010. Gender Differences in Attention-Deficit/Hyperactivity Disorder. Psychiatric Clinics of North America 33: 357.

Sherman, D.L. and Brophy, P.J. 2005. Mechanisms of axon ensheathment and myelin growth. Nat. Rev. Neurosci. 6: 683-690.

Skinner, M.D., Aubin, H.J., and Berlin, I. 2004. Impulsivity in smoking, nonsmoking, and exsmoking alcoholics. Addict. Behav. 29: 973-978. 
Spijker, S. 2011. Dissection of Rodent Brain Regions. In Neuroproteomics (ed. K.W. Li), pp. 1326. Springer.

Steel, Z. and Blaszczynski, A. 1998. Impulsivity, personality disorders and pathological gambling severity. Addiction 93: 895-905.

Van, L.L., Westenberg, P.M., and Crone, E.A. 2008. A developmental study of risky decisions on the cake gambling task: age and gender analyses of probability estimation and reward evaluation. Dev. Neuropsychol. 33: 179-196.

Wang, X., Deng, L., and Xu, X.M. 2013. Anterograde Axonal Tract Tracing. In Animals Models of Acute Neurogical Injuries II, Springer Protocols Handbooks (ed. Chen J.), pp. 461486.

Whiteside, S.P. and Lynam, D.R. 2003. Understanding the role of impulsivity and externalizing psychopathology in alcohol abuse: application of the UPPS impulsive behavior scale. Exp. Clin. Psychopharmacol. 11: 210-217. 


\section{Figure Captions}

Figure 1. Illustration of a coronal section of the rat brain at $3.2 \mathrm{~mm}$ anterior to bregma (Paxinos and Watson 1998) with injection sites for individual rats indicated by gray circles for females and black triangles for males.

Figure 2. Defined areas of measurement within the dorsolateral striatum and dorsomedial striatum from example female coronal section processed for biotinylated dextran amine histochemistry. Dashed arrows represent distance measurements from the corpus callosum and lateral ventricles. Circles represent $1 \mathrm{~mm}$ diameter circular regions of density analysis.

Figure 3. Biotinylated dextran amine staining in example female dorsal striatum at varying magnification levels.

Figure 4. Western blot data showing the effect of sex on protein levels of myelin basic protein (MBP) in the orbital frontal cortex (OFC) (A-C) and dorsal striatum (dSTR) (D-F) of male and female rats. (A) Representative blot images for MBP and the loading control $\beta$-actin in OFC samples. (B) OFC data with MBP isoforms isolated. Mean density $\times$ area $(D \times A)( \pm S E M)$ expressed relative to control $\beta$-actin protein levels. Overall main effect of sex: ${ }^{*} p<0.05$. (C) OFC data with MBP isoforms averaged. Mean $\mathrm{D} \times \mathrm{A}( \pm \mathrm{SEM})$ expressed relative to control $\beta$ actin protein levels. Overall main effect of sex: ${ }^{*} p<0.05$. (D) Representative blot images for MBP and the loading control $\beta$-actin in dSTR samples. (E) dSTR data with MBP isoforms isolated. Mean $\mathrm{D} \times \mathrm{A}( \pm \mathrm{SEM})$ expressed relative to control $\beta$-actin protein levels. $(\mathrm{F}) \mathrm{dSTR}$ data 
with MBP isoforms averaged. Mean $\mathrm{D} \times \mathrm{A}( \pm \mathrm{SEM})$ expressed relative to control $\beta$-actin protein levels.

Figure 5. Western blot data showing the effect of sex on protein levels of myelin proteolipid protein (PLP) in the orbital frontal cortex (OFC) (A-B) and dorsal striatum (dSTR) (C-D) of male and female rats. (A) Representative blot images for PLP and the loading control $\beta$-actin in OFC samples. (B) OFC data. Mean density $\times$ area $(\mathrm{D} \times \mathrm{A})( \pm$ SEM) expressed relative to control $\beta$-actin protein levels. Overall main effect of sex: $* p<0.05$. (C) Representative blot images for PLP and the loading control $\beta$-actin in dSTR samples. (D) dSTR data. Mean D×A $( \pm$ SEM) expressed relative to control $\beta$-actin protein levels.

Figure 6. (A) Biotinylated dextran amine (BDA) density in the dorsolateral striatum (dlSTR) of male and female rats across hemispheres. Overall main effect of sex: ${ }^{*} p=0.039$. (B) BDA density in the dorsomedial striatum (dmSTR) of male and female rats across hemispheres. Overall main effect of sex: ${ }^{\#} p=0.058$. 

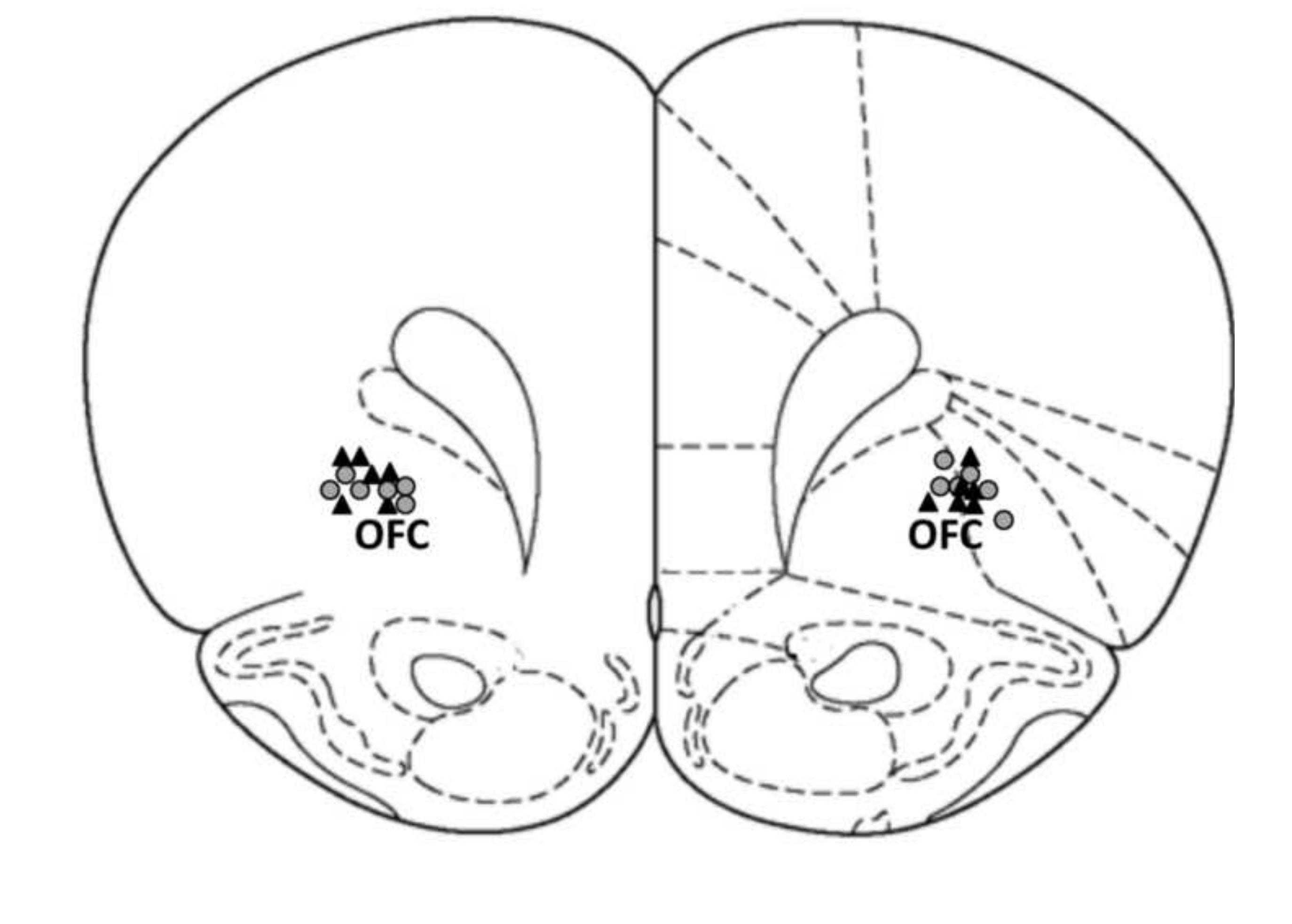


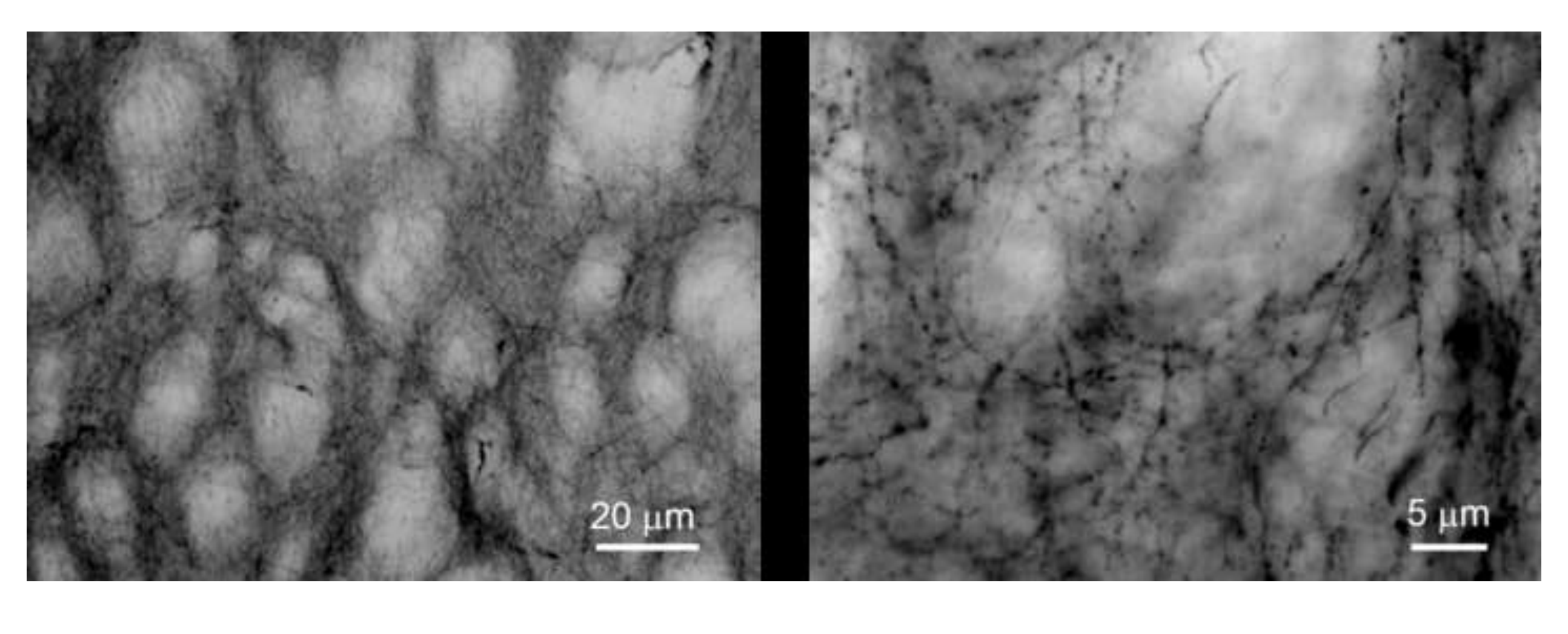

.
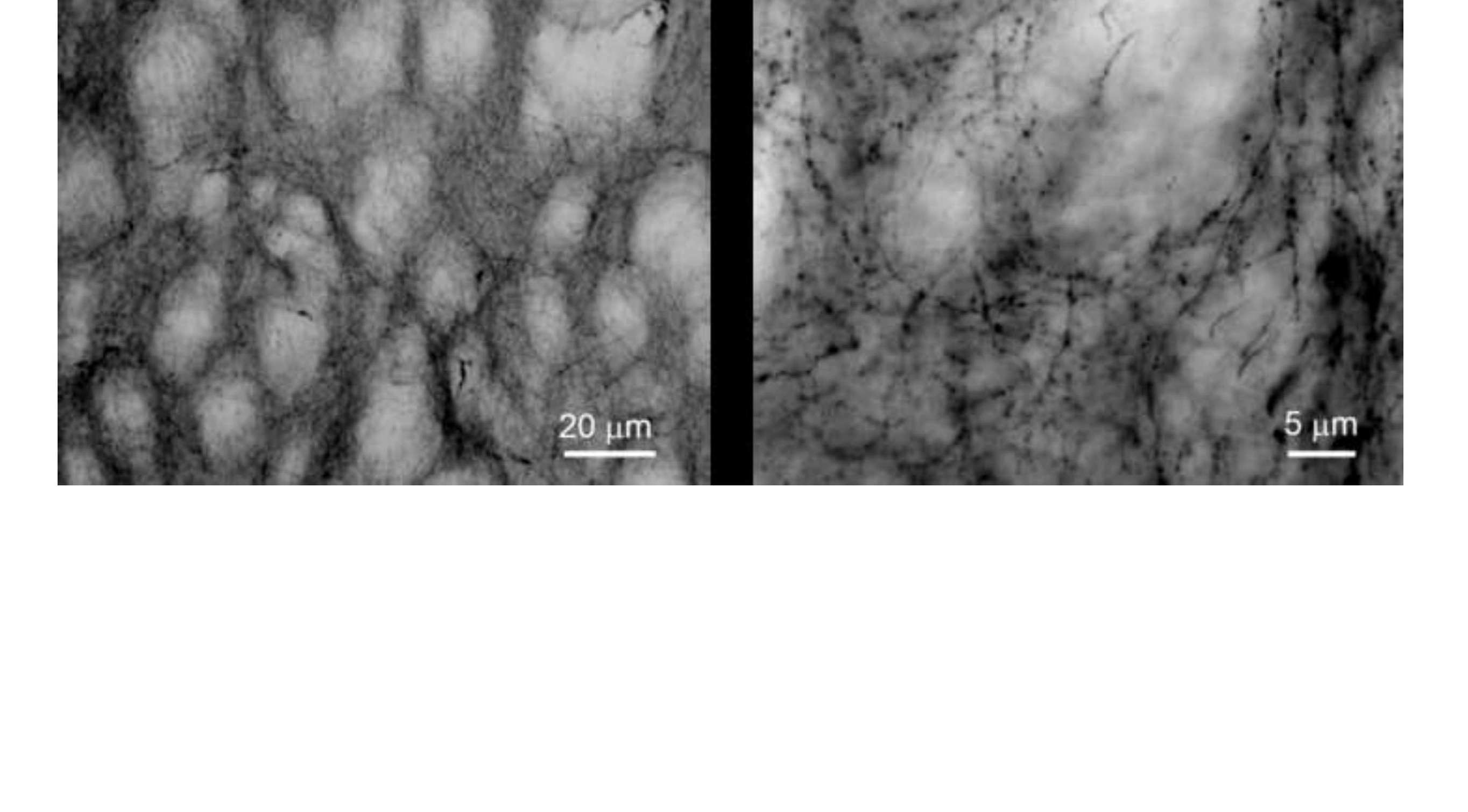

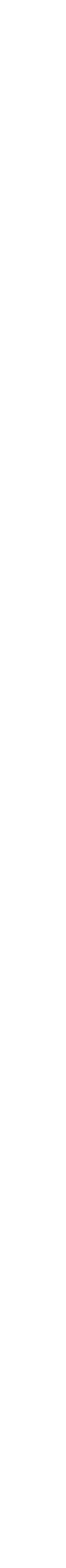




\section{OFC}

A

F M

MBP $21.5 \mathrm{kD} \rightarrow$
MBP $18.5 \mathrm{kD} \rightarrow$
$\operatorname{MBP} 17 \mathrm{kD} \rightarrow$
$\operatorname{MBP} 14 \mathrm{kD} \rightarrow$

$\beta$-actin $\longrightarrow$

B

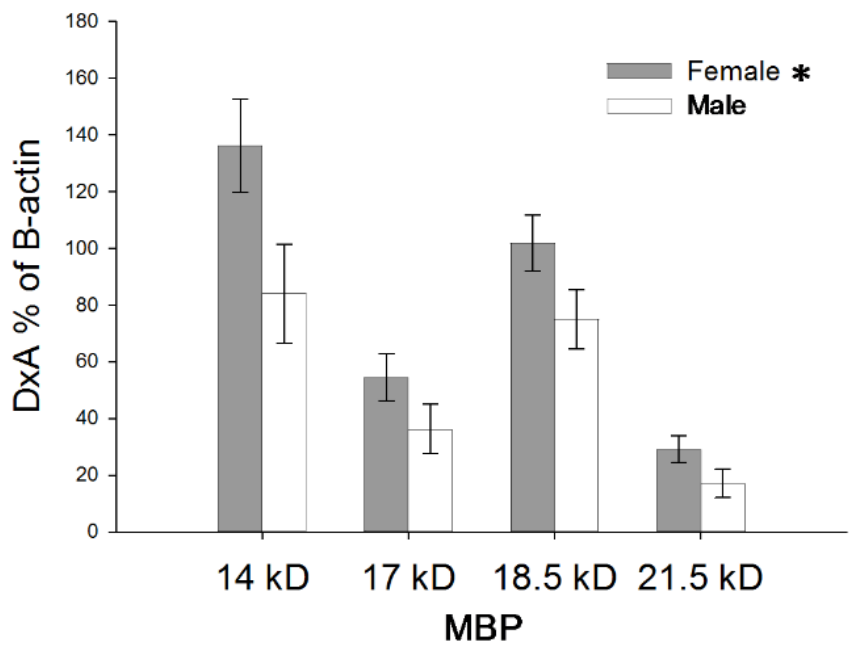

C

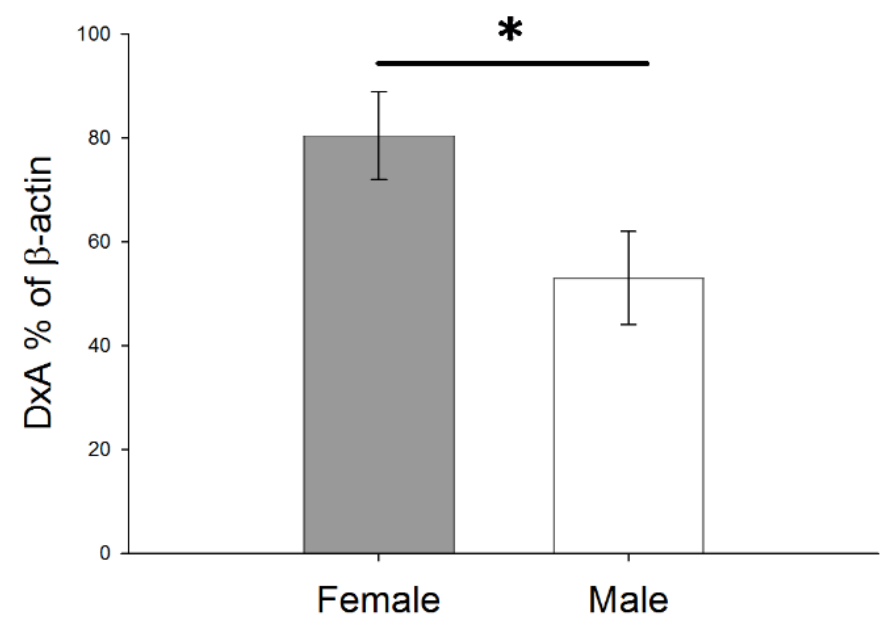

dSTR

D

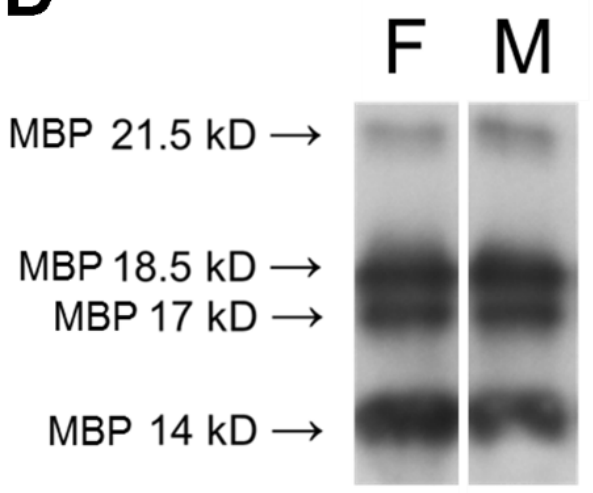

$\beta$-actin $\rightarrow$

E

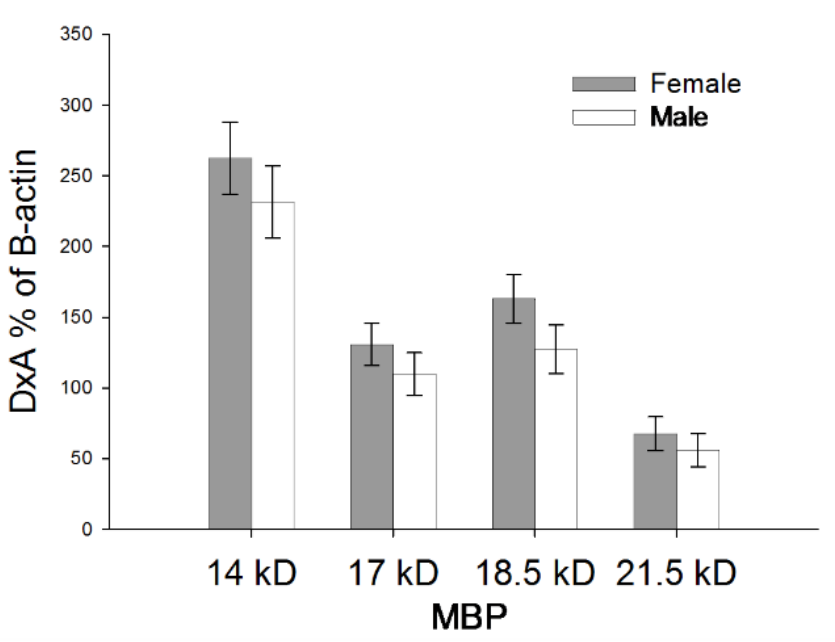

F

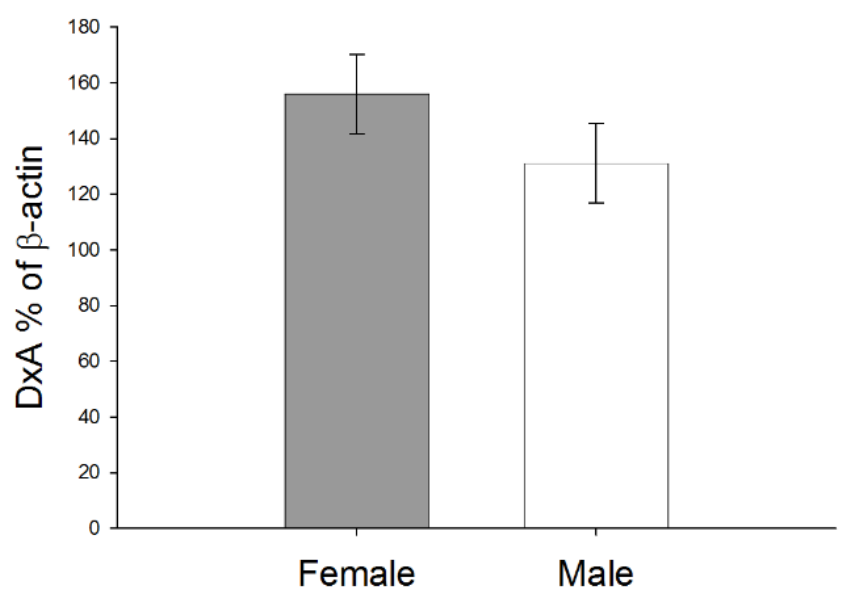




\section{OFC}

A

$$
\begin{aligned}
& \text { F M } \\
& \begin{array}{l}
\mathrm{PLP} \rightarrow=- \\
\beta \text {-actin } \rightarrow=
\end{array}
\end{aligned}
$$

B

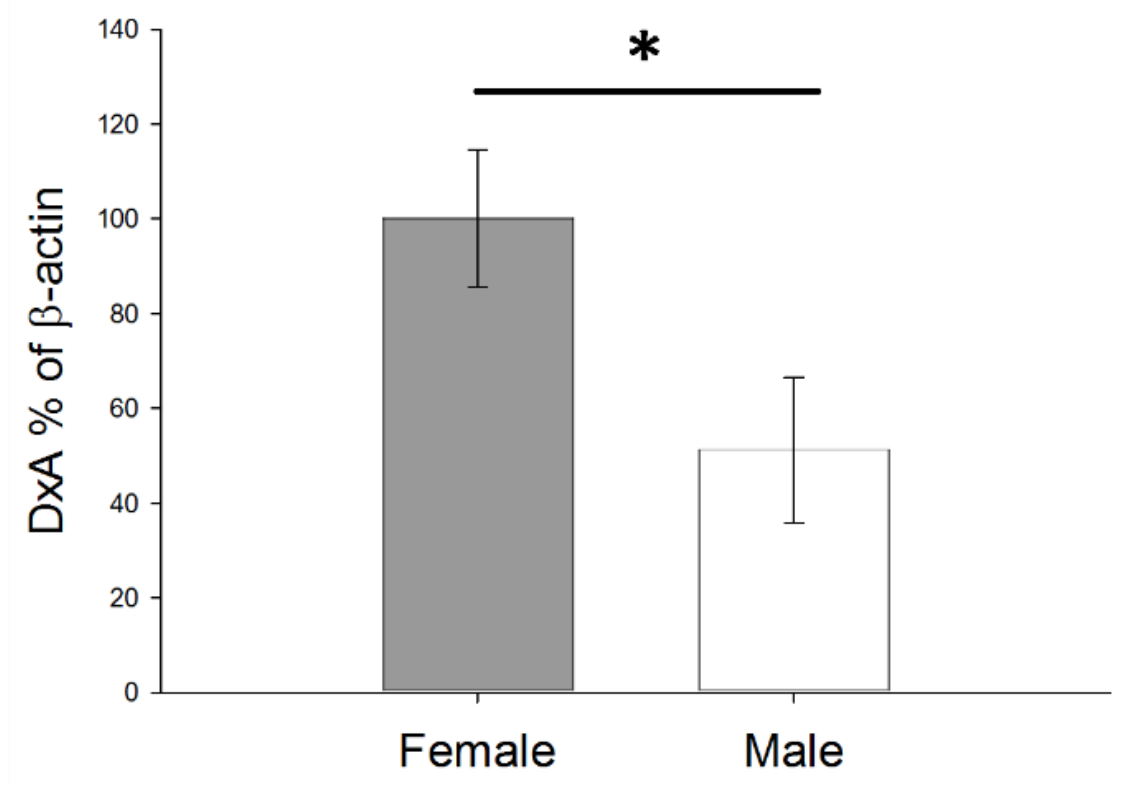

\section{dSTR}

C

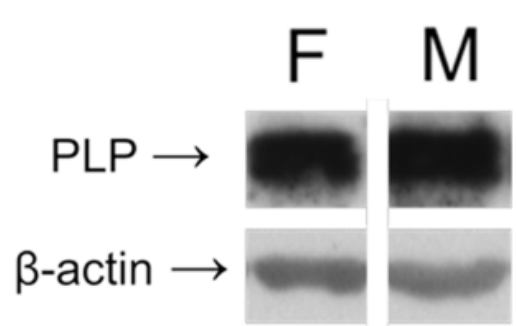

D

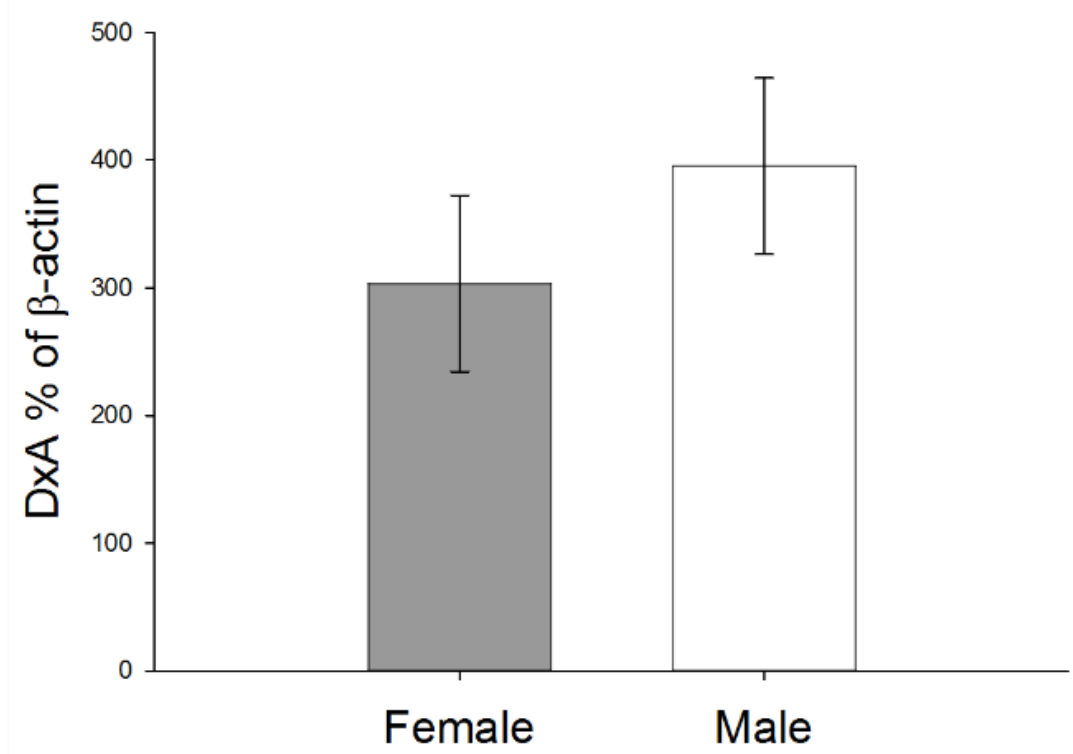


A

dISTR

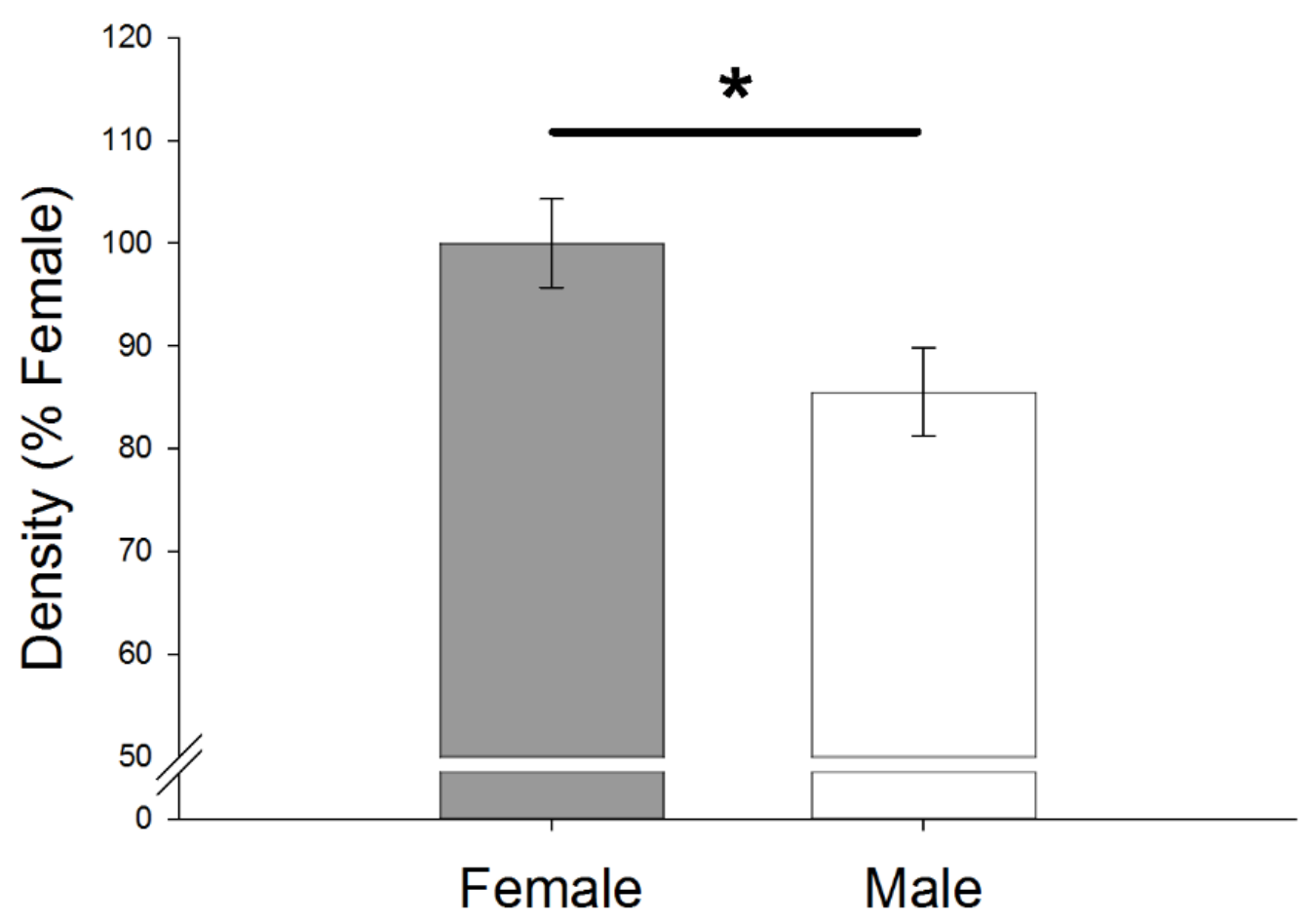

dmSTR

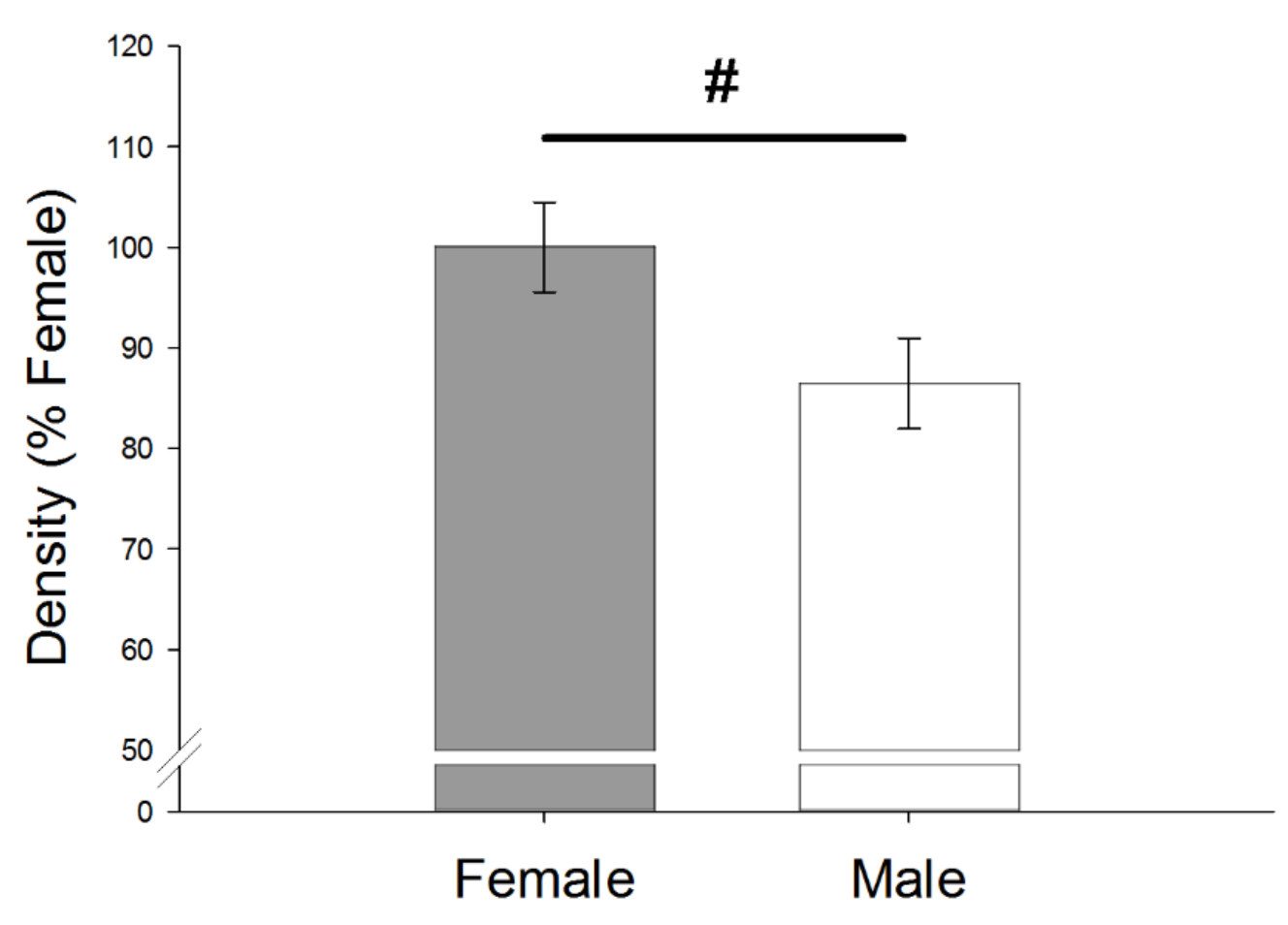

Figure 6

\section{B}

dmsTR 